\title{
Different cardiac performance in severe and mild patients with COVID-19 by standard and strain echocardiography
}

\author{
HUi Wang ${ }^{1}$, Zhiyue Liu $^{2}$, Jiafu Wei ${ }^{1}$, Jun Lin ${ }^{1}$, Yang Ge ${ }^{1}$, Huanxing Li $^{1}$, Tao Zeng ${ }^{1}$, Mei \\ Liu $^{2}$, Fangyang Huang ${ }^{1}$, Qi Liu ${ }^{1}$, Mao Chen ${ }^{1}$, and He Huang ${ }^{1}$ \\ ${ }^{1}$ Affiliation not available \\ ${ }^{2}$ Sichuan University West China Hospital
}

September 11, 2020

\begin{abstract}
Background: The initial mechanism of COVID-19 is the binding of the virus to ACE2. Since the heart and the vessels also express ACE2, they both could become targets of the virus. However, cardiac performance of mild and severe patients may be different, requested individualized treatment. The aim of this study is to explore the global and segmental myocardial performance of the severe and mild COVID-19 patients.Methods: 45 patients with COVID-19 infection were included in this study. The clinical history, laboratory test and standard and strain echocardiography were performed at admission. Results: 1. All patients showed reduced cardiac diastolic function; 2. Severe patients exhibited exacerbated right ventricular systolic function; 3. All patients showed impaired left ventricular strain, worse strain in severe patient. 4. The apical longitudinal strain of mild patients was higher than basal and middle segment. 5. There was a negative correlation between LV GLS and log TnT-hs, as well as NT-pro BNP. 6. The EF value and strain of left atrium of mild and severe patients decreased; 7. LV GLS, LV GCS and LA GLS area under the ROC curve to predict the disease severity were $0.698,0.758$ and 0.782 respectively. 8 . In the follow-up of severe patients, left atrial and ventricular strain showed an increased trend.Conclusions: These findings suggested that left ventricular performance was subclinically impaired during COVID-9 infection irrespective of infection severity and the strain of LV and LA may predict the disease severity. The cardiac function had an increasing trend for severe patients.
\end{abstract}

\section{Introduction}

The COVID-19 infection is caused by severe acute respiratory syndrome coronavirus 2 (SARS-CoV2) ${ }^{1}$. SARS-CoV2 as well as other coronaviruses have been verified to transit into their host human cells via angiotensin-converting enzyme 2 (ACE2). ACE2 is a type I integral membrane protein with many vital physiologic functions which is highly expressed in alveolar and cardiovascular cells ${ }^{2,3}$.In the published clinical studies of COVID-19, patients with acute cardiac injury ${ }^{4}$, shock, and arrhythmia were present in $7.2 \%, 8.7 \%$, and $16.7 \%$ respectively. Thus, cardiac injury has also attracted attention in COVID-19 pneumonia.

Echocardiography is the choice of method in detecting cardiac structure and function. Left ventricular global longitudinal strain (LVGLS) is beneficial for both diagnosis and risk stratification in patients with cardiac diseases $^{5}$. In the published cases, COVID-19 infected patients showed an enlarged left ventricle, diffuse myocardial dyskinesia with a decreased left ventricular ejection fraction (LVEF), pulmonary hypertension, as well as a reduced pulmonary artery systolic pressure (PASP), tricuspid annular plane systolic excursion (TAPSE) ${ }^{6}$ and a lower inferior vena cava (IVC) collapse rate. In addition, cardiac tamponade and pericardiocentesis could also happen in these patients ${ }^{7}$. Besides that, there is lack of the cohort echocardiography study to explore different cardiac performance of different severity of disease by standard and strain echocardiography in patients with COVID-19.

Therefore, this study aimed to observe 1. Different cardiac function of patients in ICU and general ward by 
standard and strain echocardiography. 2. the cardiac function change in follow-up of severe patients in ICU with COVID-19.

\section{Methods}

\subsection{Population}

45 patients diagnosed COVID-19 infection were included in our study (National Health Commission: Seventh Edition Treatment plan for COVID-19) ${ }^{8}$. Mild patients: the clinical symptoms are slight, and no pneumonia manifestations on imaging. Ordinary type: with fever, respiratory tract symptoms, etc. Imaging shows pneumonia. Severe patients: Adults with any of the following syndromes; 1 . Short of breath, RR[?] 30 times/min; 2. Oxygen saturation [?] 93\% in rest condition; 3. Arterial blood oxygen partial pressure $(\mathrm{PaO} 2)$ / oxygen concentration (FiO2) [?]300mmHg. Pulmonary imaging shows that the lesions progress significantly $>50 \%$ within 48 hours were treated as severe. Critical severity: 1 . Respiratory failure occurs and mechanical ventilation is performed; 2 . Shock occurs; 3 . Combined organ failure requires intensive care.

Among the 45 patients, nine patients have preexisting cardiac disease (six patients with hypertension, one patient with coronary heart disease and hypertension, two patients has chronic heart failure and hypertension).The clinical history, laboratory data including high-sensitivity troponin $\mathrm{T}(\mathrm{hs}-\mathrm{TnT})$, N-Terminal pro-brain natriuretic peptide (NT-pro BNP) and echocardiography data were collected at admission to the study (baseline). Because of the small study population, we divided the mild and ordinary type as mild group, 25 patients in total; severe and critical severity as severe group, 20 cases in total. The severe group in intensive care unit (ICU) were followed up for 16 days. 7 severe patients recovered and were transferred out, only 13 severe patients were still in intensive care unit and received the echocardiography at least twice(follow-up). Furthermore, 10 received echocardiography more than 7 times during hospital. The data collection was from February 22th to March 8th 2020. The first echocardiography in this study was performed on admission to the study, not at hospital admission.

23 normal people were included based on normal echocardiography and blood test during the March 2020 in west china hospital.

\subsection{Standard Echocardiographic Examination}

Transthoracic echocardiographic examinations were performed with S5-1 probe, 1-5MHz (CX50, Phillip, Netherlands). Two-dimensional (2D) and Doppler echocardiography were performed according to the guidelines of the American Society of Echocardiography ${ }^{5}$. The following echocardiographic views were recorded over three cardiac cycles: left parasternal long- and short axis views and apical two- apical three- and apical four-chamber views. Images were recorded on external hard disk and stored in digital format for offline analysis (Q-lab 10.8.5). The right ventricular anteroposterior diameter and LV diameter, left atrial (LA) diameter, LV interventricular septum (IVS) and LV posterior wall (LVPW) thickness and aortic diameter were measured at long axis view. The right atrial (RA) diameter was measured at apical four-chamber view. The pulmonary diameter was measured at pulmonary long axis view. LV systolic function included LV volumes, left ventricular ejection fraction (LVEF) were measured by the modified biplane Simpson's rule. Mitral, aortic and pulmonary artery Doppler flow profiles were recorded, including maximum mitral inflow velocity at early (E) and late (A) diastole. Pulsed tissue Doppler imaging was obtained with the sample volume placed at the septum of mitral valve and free wall of tricuspid valve at the apical 4-chamber view. Velocity of mitral annulus at early diastole (e'), late diastolic myocardial a') velocities, and RV TDI-s were recorded. The E/e' ratio, being recorded at the level of the septum of the mitral annulus, was used as an index of LV filling pressure. Tricuspid annular plane systolic excursion (TAPSE) was measured in apical four-chamber view via placing the cursor at the tricuspid anterior annulus and measuring the distance of systolic annular RV excursion along a longitudinal line. All images were collected by an experienced investigator in echocardiography who was blinded to the clinical information.

\subsection{Strain echocardiography examination}


The images were acquired at the left parasternal short axis views, apical long axis, apical four-chamber, and apical two-chamber views. All off-line analyses were performed by an investigator experienced in speckle tracking analysis using the Q-lab workstation version 10.8.5(Phillip Medical Systems). The investigator was blinded to the patient's management and outcome information. The endocardium was defined by manual tracing and the region of interest (ROI) was adjusted to the compact myocardium thickness. Default settings for smoothing and drift compensation were used. We evaluated longitudinal and circumferential strain. LV longitudinal strain analysis was performed on apical view. Circumferential strain was performed on shortaxis views of three segments; basal, mid and apex.Care was taken to ensure that the basal short-axis at the level of mitral valve, and the apical plane distal to the papillary muscle. The region of interest width was adjusted first, to include the entire endocardium. From the analysis, we received 18 regional longitudinal strain (RLS) and circumferential strain (RCS). These allowed us to calculate the segmental longitudinal and circumferential strain and the global longitudinal strain (GLS) and circumferential strain (GCS) as well. Using the same software, the LA endocardial border was traced in the apical 4-chamber view and apical 2-chamber view, care was taken to exclude the appendage and pulmonary veins from the LA cavity. Then, a composite LA longitudinal strain (LAGLS) curve throughout the cardiac cycle was generated. The frame rate of $58 \pm 3$ frames/sec was obtained for the LV and LA strain analysis.

\subsection{Statistical analysis}

Quantitative data are presented as Mean $\pm \mathrm{SD}$, qualitative data as frequency number (\%). Comparisons among the groups were performed by one-way ANOVA. If there was a difference, then S-N-K test was used. T-test was used to compare between two groups. Chi-square test was used to compare binary variables. Since values of NT-pro BNP and hs-TnT were not normally distributed, data were log-transformed for multivariate analyses. Pearson's correlation was used to test the association between LV GLS and log hs-TnT, as well as NT-pro BNP. The Receiver Operating Characteristic (ROC) curve was performed to evaluate the GLS's and GCS's ability to predict the severity of the disease. Area under the curve(AUC) $<0.5$ has no diagnostic value, $0.5<\mathrm{AUC}<0.7$ has low diagnostic value, $0.7<\mathrm{AUC}<0.9$ has medium diagnostic value, and $\mathrm{AUC}>0.9$ has high diagnostic value. ${ }^{9}$ when AUC $>0.9$, according to Youden Index, combining sensitivity and specificity, find the best diagnostic cut point. $P<0.05$ was considered significant. All statistical tests were analyzed with SPSS (version 19.0) software.

\section{Results}

\subsection{The cardiac structure and function in patients with COVID-19 at baseline}

The mean age of the patients with COVID-19 was 55.33+-19.65 years old, with a range from 20 to 87 . There were 23 men and 22 women in this study. Nine patients had hypertension, in whom one had coronary heart disease and two had chronic heart disease. (Table 1)

None of the patients had significant valvular lesions. Trace to mild tricuspid regurgitation was found in 8 mild patients (32\%), and in 6 severe patients (30\%). No significant pulmonary hypertension was found. Table 2 showed the echocardiographic data at mild and severe patients. Significantly thicker interventricular septum (IVS) $(P<0.05)$ and posterior wall of left ventricle $(\mathrm{LVPW})(P<0.05)$, higher $\mathrm{E} / \mathrm{e}^{\prime}(P<0.05)$, were observed in severe patients when compared with control group, IVS and LVPW was thicker $(P<0.05)$ in severe patients than in mild patients. E/e' was larger in mild group than controls. $(P<0.05)$.

larger right atrium $(P<0.05)$, lower TAPSE $(P<0.05)$ and TDI-s $(P<0.05)$ were observed in severe patients when compared with control group. The size of RA was larger in mild group than controls. $(P<0.05)$. Moreover, TDI-s was significantly lower $(P<0.05)$ in the group of severe patients than in mild patients. LAEF decreased in patients with COVID-19 and no difference in LAESV among these three groups.

\subsection{Left atrial strain and left ventricular strain in patients with COVID-19 at baseline}

GLS and GCS patterns of left ventricle and GLS of left atrium during the cardiac cycle were reported in Table 3. LA GLS in severe and mild patients with COVID-19 were lower than $\operatorname{controls}(P<0.05)$. LV 
GLS, LV GCS and LA GLS in severe and mild patients with COVID-19 were lower than controls $(P<0.05)$. LVGLS, LVGCS in severe patients were lower than mild patients $(P<0.05)$.

Basal, middle and apical segment of circumferential strain (CS) and longitudinal strain (LS) in severe and mild patients were presented in Table 4. No significant difference was observed among basal, middle and apical segments in LV CS and LV LS in severe group. No difference in three segments in LV CS in mild patients, however, apical LS was higher than LS in the other two segments in mild patients.

In severe patients with COVID-19(n=20), the value of $\log (\mathrm{NT}$-pro BNP) and $\log (\mathrm{hs}-\mathrm{TnT})$ were 2.50+-0.94 $\mathrm{pg} / \mathrm{ml}$ and $1.12+-0.57 \mathrm{pg} / \mathrm{ml}$ respectively. There was a negative correlation between LV GLS and $\log$ TnT-hs as well as NT pro-BNP in severe patients with COVID -19 (figure 1).

We analyzed the relation between the severity of COVID-19 patients and LV GLS, LV GCS and LA GLS through ROC curve and defined the mild condition as positive status (Figure2). The results showed that the URC of LV GLS, LV GCS and LA GLS to predict the severity were 0.698(0.543-0.826), 0.758(0.607-0.873) and 0.782(0.634-0.891) respectively. LV GLS, LV GCS and LA GLS may had a low or medium diagnostic performance for the severity of COVID-19 patients (all $P<0.05)$.

\subsection{The cardiac function in severe patients with COVID-19 during follow-up}

There was no statistical difference in the size of both atria and ventricle, thickness of interventricular septum, left ventricular posterior wall, diameter of aorta and pulmonary between the baseline and follow-up results. No difference was also observed in left ventricular systolic function (LVEF) and diastolic function (E/e'), LAEF, IVC collapse rate and systolic (TAPSE, TDI-S) function of right ventricle. (Table 5)

The GCS of left ventricle was significantly higher $(P<0.05)$ at follow-up when compared with baseline. However, there was no statistical difference in LV GLS of severe patients at baseline and follow-up, neither in LA GLS (Table 6).

Only 10 severe patients received echocardiography examination more than 7 times during hospitalization. The patient number was small, thus we drew a line chart to show the strain in left atrium and left ventricle. The results showed that the LA GLS, LV GCS and LV GLS tended to rise gradually. (Figure 3).

\section{Discussion}

Acute myocardial injury has been demonstrated in $7.2 \%-12 \%$ of patients with COVID-19 in preliminary reports, with a higher prevalence among those requiring intensive care ${ }^{10}$. Mortality data from 44672 cases of COVID-19 released by the Chinese Centre for Disease Control and Prevention demonstrate that patients with cardiovascular comorbidities show a much higher mortality ${ }^{11}$.

But there still lack a systematic and comprehensive study including mild and severe patients in the assessment of left ventricular, left atrial and right ventricular function.

The aim of the present study was to evaluate the cardiac function by standard and strain echocardiography including mild and severe patients with COVID-19. We observed that: 1. Both mild and severe COVID-19 infected patients showed reduced left ventricular diastolic function compared with control group; 2. Severe patients with COVID-19 exhibited exacerbated right ventricular systolic function; 3. Both mild and severe patients with COVID-19 showed impaired left ventricular strain, and the strain in severe patients even worse, suggesting all the patients may had early systolic function. 4 . The strain in apical segment of mild patients with COVID-19 was elevated compared with basal and middle segment. 5.There was a negative correlation between LV GLS and log TnT-hs, as well as NT-pro BNP. 6. The EF value and strain of left atrium of mild and severe patients with COVID-19 decreased; 7.LV GLS, LV GCS and LA GLS might predict the severity of COVID-19. 8. In the follow-up of severe COVID-19 infected patients, their cardiac structure and function had no change, while left atrial and ventricular strain exhibited an increased trend.

In the present study, there was no difference in the size of LV and LVEF, among the 3 groups. IVS and LVPW in severe patients with COVID-19 were thicker than mild patients and the controls. There were 8 
severe patients with hypertension. However, only one mild patient with hypertension. That might contribute to thicker IVS and $\mathrm{LVPW}^{12}$. In addition, the age may also have an effect on this cardiac performance ${ }^{13}$. In our investigation, E/e' in severe patients with COVID-19 were higher than mild and control groups, probably suggesting elevated LV filling pressure. Furthermore, no difference in RV among the 3 groups. TDI-s and TAPSE, the index of right ventricular systolic function, in severe patients were lower than that in the other two groups, suggesting right ventricular systolic dysfunction, which was seen, particularly in the context of severe parenchymal lung disease and acute respiratory disease ${ }^{13}$. Previous study also demonstrated that compared with survivors, nonsurvivors displayed enlarged right heart chambers, diminished RV function, and elevated pulmonary artery systolic pressure. ${ }^{14} 7$ of $17(42 \%)$ needed prone positioning for severe Hypoxia had RV dysfunction(RV FAC $<35 \%$ ). ${ }^{15}$

LA is considered to modulate left ventricular filling and cardiovascular performance as (i) a reservoir for pulmonary venous return during ventricular systole,(ii) a conduit for pulmonary venous return during early ventricular diastole, and (iii) a booster pump that augments ventricular filling during late ventricular diastole. ${ }^{16} \mathrm{LA}$ size is strong predictor of cardiovascular morbidity and death ${ }^{17}$. LA size correlates with both LA and left ventricular(LV) function. Previous study found no difference in LA dimension between survivors and nonsurvivors with COVID- $19^{14}$, and there is still lack of study on left atrial strain for now. In our study, higher LA volume and lower LAEF were observed in severe and mild patients with COVID-19 than control group. Further, LA myocardial deformation is assessed as global longitudinal strain. ${ }^{18} \mathrm{In}$ addition, the results showed that LAGLS decreased in severe and mild patients, suggesting the LA systolic dysfunction. All LA volume, LAEF and LAGLS showed LA systolic dysfunction, contributing to LV dysfunction.

Strain image is superior to standard echocardiography for myocardial injury detection in patients and reflects the complex deformation pattern of the heart during systole ${ }^{19}$. Previous study showed that LV GLS measurement are stable and repeatable, which have additional predictive value for evaluating the rest cardiac function of patients. ${ }^{20}$ The normal LV GLS measured by Qlab is $18.9+-2.5 \%{ }^{21}$. Though there was no significant change in LVEF and LV volumes, significantly lower LV GLS and LV GCS were observed in severe and mild patients with COVID-19 compared with control group, especially in severe group. Although in severe patients, 2 patients with coronary heart disease and 1 with chronic heart disease may contribute to worse GLS. Patients with hypertension may also lead to lower GLS ${ }^{22}$. This result showed subclinical cardiac systolic dysfunction in patients with COVID-19. Troponin and NT-pro BNP is a widely accepted biomarker of myocardial injury, and elevated serum levels have been a notable feature during recent epidemics of respiratory virus infections. ${ }^{20}$ Accordingly, we observed the troponin and NT-pro BNP were negatively correlated with LV GLS in severe patients. Previous data indicated a decreased myocardial longitudinal strain in fulminat myocarditis especially decreased in the basal and middle segments ${ }^{4,23}$, which was similar to our results, our results observed the apical LS in mild patients was higher than middle and basal segment, however, no significant difference among basal, middle and apical LS,CS in severe patients, as shown in table 4. Previous study showed that GLS can predict functional capacity in patients with preserved LVEF heart failure and to assess prognosis in reduced LVEF heart failure ${ }^{24}$. And it could classify HF patients according to the functional capacity. ${ }^{25}$ In this study, we found that GLS had a low predicted value of the disease severity, which may stratify the patients with unknown condition and therefore to deserve more differentiated treatment.

In our study, only 13 severe patients in ICU received echocardiography one more time. Compared with the first examination, no change in cardiac structure on follow-up. For the strain, our results showed only LV GCS increased $(30.40+-7.51$ vs $23.05+-8.56, P<0.05)$ in severe patients when compared with the first echocardiography, while the LV GLS and LA GLS exhibited an increased trend. Furthermore, the line chart based on 10 patients for 16 days also showed steadily rising trend. Alleviating myocardial injury was implied, although which needed longer observation. Previous study showed cardiac impairment caused by SARS-CoV in the more critically ill patients may be reversible on recovery ${ }^{26}$.

\section{Study Limitations}

Outbreak of COVID-19 was abrupt, the study was an observational, 'not well-designed' one in highly contagious COVID-19 patients, lasting only 16 days. The study population number in our study was small. 


\section{Conclusions}

These findings suggested that left ventricular performance was subclinically impaired during COVID-9 infection irrespective of infection severity and the strain of LV and LA may predict the disease severity. The cardiac function had an increasing trend for severe patients treated in ICU. Whether the myocardial injury may be reversible on clinical recovery needs longer follow-up.

\section{Acknowledgements}

We thank the COVID-9 Clinical Taskforce, Sichuan Province, and all the health care providers and institutions involved in the management of patients with COVID-9.

\section{Disclosures}

All authors declare that they have no conflict of interest to disclose.

ORCID https://orcid.org/0000-0001-9192-1419

\section{References}

1. Huang C, Wang Y, Li X, et al. Clinical features of patients infected with 2019 novel coronavirus in Wuhan, China. Lancet (London, England) 2020;395:497-506.

2. Zhou P, Yang XL, Wang XG, et al. A pneumonia outbreak associated with a new coronavirus of probable bat origin. Nature 2020;579:270-3.

3. Ge XY, Li JL, Yang XL, et al. Isolation and characterization of a bat SARS-like coronavirus that uses the ACE2 receptor. Nature 2013;503:535-8.

4. Sala S, Peretto G, Gramegna M, et al. Acute myocarditis presenting as a reverse Tako-Tsubo syndrome in a patient with SARS-CoV-2 respiratory infection. European heart journal 2020;41:1861-2.

5. Lang RM, Badano LP, Mor-Avi V, et al. Recommendations for cardiac chamber quantification by echocardiography in adults: an update from the American Society of Echocardiography and the European Association of Cardiovascular Imaging. Journal of the American Society of Echocardiography : official publication of the American Society of Echocardiography 2015;28:1-39.e14.

6. Zeng JH, Liu YX, Yuan J, et al. First case of COVID-19 complicated with fulminant myocarditis: a case report and insights. Infection 2020:1-5.

7. Hua A, O'Gallagher K, Sado D, et al. Life-threatening cardiac tamponade complicating myo-pericarditis in COVID-19. European heart journal 2020.

8. National Health Commission of the People's Republic of China. Novel Coronavirus-Pneumonia diagnostic and treatment regimens. Available:http://www. nhc. gov. cn/yzygj/ s7653p/ new_ list. shtml.

9. Hanley JA, McNeil BJ. The meaning and use of the area under a receiver operating characteristic (ROC) curve. Radiology 1982;143:29-36.

10. Huang C, Wang Y, Li X, et al. Clinical features of patients infected with 2019 novel coronavirus in Wuhan, China. The Lancet 2020;395:497-506.

11. Devereux RB, Liebson PR, Horan MJ. Recommendations concerning use of echocardiography in hypertension and general population research. Hypertension 1987;9:Ii97-104.

12. Huwez FU, Houston AB, Watson J, et al. Age and body surface area related normal upper and lower limits of M mode echocardiographic measurements and left ventricular volume and mass from infancy to early adulthood. British heart journal 1994;72:276-80. 
13. Driggin E, Madhavan MV, Bikdeli B, et al. Cardiovascular Considerations for Patients, Health Care Workers, and Health Systems During the COVID-19 Pandemic. Journal of the American College of Cardiology 2020;75:2352-71.

14. Li Y, Li H, Zhu S, et al. Prognostic Value of Right Ventricular Longitudinal Strain in Patients With COVID-19. JACC Cardiovascular imaging 2020.

15. Beyls C, Bohbot Y, Huette P, et al. Tricuspid Longitudinal Annular Displacement for the Assessment of Right Ventricular Systolic Dysfunction during Prone Positioning in Patients with COVID-19. Journal of the American Society of Echocardiography : official publication of the American Society of Echocardiography 2020 .

16. Donal E, Behagel A, Feneon D. Value of left atrial strain: a highly promising field of investigation. Eur Heart J Cardiovasc Imaging 2015;16:356-7.

17. Hoit BD. Left atrial size and function: role in prognosis. Journal of the American College of Cardiology 2014;63:493-505.

18. Badano LP, Kolias TJ, Muraru D, et al. Standardization of left atrial, right ventricular, and right atrial deformation imaging using two-dimensional speckle tracking echocardiography: a consensus document of the EACVI/ASE/Industry Task Force to standardize deformation imaging. Eur Heart J Cardiovasc Imaging 2018;19:591-600.

19. Stoylen A, Slordahl S, Skjelvan GK, et al. Strain rate imaging in normal and reduced diastolic function: comparison with pulsed Doppler tissue imaging of the mitral annulus. Journal of the American Society of Echocardiography : official publication of the American Society of Echocardiography 2001;14:264-74.

20. Alhogbani T. Acute myocarditis associated with novel Middle east respiratory syndrome coronavirus. Ann Saudi Med 2016;36:78-80.

21. Lang RM, Badano LP, Mor-Avi V, et al. Recommendations for cardiac chamber quantification by echocardiography in adults: an update from the American Society of Echocardiography and the European Association of Cardiovascular Imaging. European Heart Journal-Cardiovascular Imaging 2015;16:233-71.

22. Kim SA, Park SM, Kim MN, et al. Assessment of Left Ventricular Function by Layer-Specific Strain and Its Relationship to Structural Remodelling in Patients With Hypertension. The Canadian journal of cardiology 2016;32:211-6.

23. Fei,MIAO ZH-jZNJJ-gCCLRM, Hong KWD-wW. Evaluation of left ventricular function in mild myocarditis and fulminant myocarditis patients by two-dimensional speckle-tracking echocardiography. Journal of critical and severe internal medicine 2018:451-5.

24. Hasselberg NE, Haugaa KH, Sarvari SI, et al. Left ventricular global longitudinal strain is associated with exercise capacity in failing hearts with preserved and reduced ejection fraction. Eur Heart J Cardiovasc Imaging 2015;16:217-24.

25. Maia RJC, Brandao SCS, Leite J, et al. Global Longitudinal Strain Predicts Poor Functional Capacity in Patients with Systolic Heart Failure. Arquivos brasileiros de cardiologia 2019;113:188-94.

26. Li SS, Cheng CW, Fu CL, et al. Left ventricular performance in patients with severe acute respiratory syndrome: a 30-day echocardiographic follow-up study. Circulation 2003;108:1798-803.

\section{Figure legends}

Figure 1. a. Correlation between LV GLS and $\log$ hs-TnT; b. Correlation between LV GLS and $\log$ NT-pro BNP. ( $\mathbf{n = 2 0}$ in each group).hs-TnT, high-sensitivity toponin T; NT-pro BNP, N-Terminal pro-brain natriuretic peptide; LV, left ventricular; GLS global longitudinal strain; 
Figure2. LV GLS - LV GCS an LA GLS as a predictor of severity of the COVID-19 patients. LV, left ventricular; LA, left atrial; GLS, global longitudinal strain; GCS, global circumferential strain.

Figure3. The change of LAEF(a), LA GLS(b), LV GLS(c) and LV GCS(d) post onset. LV, left ventricular; LA, left atrial; EF, ejection fraction; GLS, global longitudinal strain; GCS, global circumferential strain.

Tables

Table 1. Study population

\begin{tabular}{|c|c|c|c|c|}
\hline & Control(n=23) & $\operatorname{Mild}(\mathrm{n}=25)$ & Severe $(n=20)$ & $P$ value \\
\hline Male (\%) & $13(57)$ & $13(52)$ & $10(50)$ & 0.91 \\
\hline Age (years old) & $42.17 \pm 14.72 \quad 20^{\sim} 70$ & $48.44 \pm 16.1020^{\sim} 82$ & $\begin{array}{l}63.95 \pm 20.64^{*} \# \\
20^{\sim} 87\end{array}$ & $<0.001$ \\
\hline $\begin{array}{l}\text { Duration of } \\
\text { observation(days) }\end{array}$ & & $8 \pm 9.1$ & $8.1 \pm 6.2$ & 0.661 \\
\hline $\mathrm{SBP}(\mathrm{mmHg})$ & $123.17 \pm 14.21$ & $124.92 \pm 15.72$ & $141.55 \pm 18.70^{*} \#$ & 0.014 \\
\hline DBP (mmHg) & $79.61 \pm 10.22$ & $81.42 \pm 7.90$ & $79.82 \pm 13.88$ & 0.717 \\
\hline Hypertension & 0 & 1 & 8 & \\
\hline $\begin{array}{l}\text { Coronary heart } \\
\text { disease }\end{array}$ & 0 & 0 & 1 & \\
\hline $\begin{array}{l}\text { Chronic heart } \\
\text { failure }\end{array}$ & 0 & 0 & 2 & \\
\hline
\end{tabular}

SBP indicates systolic blood pressure; DBP diastolic blood pressure. ${ }^{*}$ compared with control group,$P<$ 0.05; \# compared with mild patients, $P<0.05$.

Table 2. Standard echocardiography at baseline in patients with COVID-19

\begin{tabular}{lllll}
\hline & Control $(\mathrm{n}=23)$ & Mild $(\mathrm{n}=25)$ & Severe $(\mathrm{n}=20)$ & $P$ value \\
\hline LV $(\mathrm{mm})$ & $40.04 \pm 3.80$ & $40.88 \pm 3.30$ & $41.30 \pm 10.35$ & 0.78 \\
LA (mm) & $29.70 \pm 2.80$ & $30.92 \pm 3.01$ & $29.80 \pm 3.81$ & 0.35 \\
RV (mm) & $20.87 \pm 1.78$ & $20.24 \pm 2.09$ & $20.10 \pm 3.33$ & 0.53 \\
RA (mm) & $33.57 \pm 3.27$ & $38.80 \pm 4.51^{*}$ & $37.95 \pm 4.71^{*}$ & $<0.001$ \\
IVS (mm) & $8.43 \pm 1.73$ & $8.96 \pm 1.86$ & $10.70 \pm 2.08^{*} \#$ & 0.001 \\
LVPW (mm) & $7.87 \pm 0.69$ & $7.72 \pm 1.72$ & $8.95 \pm 1.47^{*} \#$ & 0.009 \\
AAO (mm) & $29.70 \pm 2.80$ & $29.68 \pm 4.30$ & $29.55 \pm 3.30$ & 0.98 \\
PA (mm) & $19.26 \pm 2.07$ & $20.40 \pm 1.63$ & $19.70 \pm 2.89$ & 0.20 \\
LV EDV (ml) & $83.35 \pm 22.94$ & $85.70 \pm 28.09$ & $87.56 \pm 14.07$ & 0.44 \\
LV ESV (ml) & $32.78 \pm 12.49$ & $29.52 \pm 5.16$ & $28.50 \pm 13.97$ & 0.21 \\
LVEF (\%) & $69.12 \pm 6.85$ & $69.12 \pm 6.85$ & $61.96 \pm 5.42$ & 0.23 \\
AV $(\mathrm{m} / \mathrm{s})$ & $1.27 \pm 0.17$ & $1.23 \pm 0.27$ & $1.28 \pm 0.20$ & 0.75 \\
E $(\mathrm{m} / \mathrm{s})$ & $0.76 \pm 0.16$ & $0.69 \pm 0.19$ & $0.67 \pm 0.25$ & 0.27 \\
e'(cm/s) & $10.52 \pm 3.00$ & $8.04 \pm 3.26^{*}$ & $6.75 \pm 3.02^{*}$ & 0.001 \\
E/ e' & $7.57 \pm 2.21$ & $9.57 \pm 3.82^{*}$ & $11.53 \pm 1.42^{*}$ & 0.015 \\
TAPSE (mm) & $22.43 \pm 3.06$ & $21.04 \pm 2.95$ & $20.30 \pm 3.25^{*}$ & 0.03 \\
TDI-s (cm/s) & $13.61 \pm 2.53$ & $14.28 \pm 2.70$ & $11.85 \pm 5.51^{*} \#$ & 0.032 \\
LAEF $(\%)$ & $67.9 \pm 8.06$ & $47.73 \pm 7.84^{*}$ & $42.72 \pm 2.58^{*}$ & $<0.001$ \\
LA ESV (ml) & $36.56 \pm 13.91$ & $28.35 \pm 7.53$ & $30.15 \pm 14.96$ & 0.536 \\
LA EDV (ml) & $5.69 \pm 2.29$ & $14.65 \pm 4.06^{*}$ & $17.88 \pm 11.02^{*}$ & 0.002 \\
\hline
\end{tabular}


LV indicates left ventricular; LA left atrium; RV right ventricular; RA right atrium; IVS interventricular septum; LVPW left ventricular posterior wall; AAO ascending aorta; PA pulmonary artery; EDV enddiastolic volume; ESV end-systolic volume; EF ejection fraction; AV aortic blood flow velocity; E peak mitral inflow early filling velocity; e', early diastolic myocardial velocity; TAPSE Tricuspid annulus systolic displacement; TDI-s early systolic myocardial velocity; ${ }^{*}$ compared with control group,$P<0.05$; \# compared with mild patients, $P<0.05$.

\section{Table 3 LAGLS, LVGCS, LV GLS by strain echocardiography}

\begin{tabular}{lllll}
\hline & Control $(\mathrm{n}=23)$ & Mild $(\mathrm{n}=25)$ & Severe $(\mathrm{n}=20)$ & $P$ value \\
\hline LA GLS & $40.13 \pm 10.09$ & $18.83 \pm 6.32^{*}$ & $14.88 \pm 6.23^{*}$ & $<0.001$ \\
LV GCS & $37.43 \pm 5.95$ & $27.13 \pm 4.51^{*}$ & $21.06 \pm 8.78^{* \#}$ & $<0.001$ \\
LV GLS & $20.43 \pm 3.50$ & $18.79 \pm 3.73^{*}$ & $15.82 \pm 4.39^{* \#}$ & $<0.001$ \\
\hline
\end{tabular}

LV indicted left ventricular; LA left atrium; EDV end-diastolic volume; ESV end-systolic volume; EF ejection fraction; GLS global longitudinal strain; GCS global circumferential strain. * compared with control group ,$P<0.05$; \# compared with mild patients, $P<0.05$.

Table 4 Regional LV strains

\begin{tabular}{lllll}
\hline & Basal segment & Middle segment & Apical segment & $P$ Value \\
\hline LV RCS (severe) & $16.29 \pm 8.06$ & $22.49 \pm 10.55$ & $24.39 \pm 11.31$ & 0.082 \\
LV RLS (severe) & $14.07 \pm 3.82$ & $15.72 \pm 5.24$ & $17.68 \pm 6.11$ & 0.170 \\
LV RCS (mild) & $25.55 \pm 6.49$ & $27.47 \pm 5.68$ & $28.38 \pm 5.13$ & 0.297 \\
LV RLS (mild) & $17.11 \pm 5.25$ & $17.77 \pm 4.58$ & $21.49 \pm 4.90^{*} \#$ & 0.015 \\
\hline
\end{tabular}

LV indicted left ventricular; RLS regional longitudinal strain; RCS regional circumferential strain. * compared with basal segment, $P<0.05$; \# compared with middle segment, $P<0.05$.

Table 5 Echocardiographic Data at Baseline and Follow-Up in severe patients with COVID-19

\begin{tabular}{llll}
\hline & Baseline $(\mathrm{n}=13)$ & Follow-up(n=13) & $P$ value \\
\hline LV $(\mathrm{mm})$ & $45.62 \pm 4.234$ & $44.77 \pm 6.57$ & 0.422 \\
LA (mm) & $30 \pm 4.22$ & $32 \pm 2.80$ & 0.128 \\
RV (mm) & $20.46 \pm 3.38$ & $20.00 \pm 3.03$ & 0.475 \\
RA (mm) & $36.92 \pm 4.31$ & $35.00 \pm 12.0$ & 0.505 \\
IVS (mm) & $11.31 \pm 1.80$ & $11.31 \pm 1.80$ & 1 \\
LVPW (mm) & $9.31 \pm 1.65$ & $9.62 \pm 1.61$ & 0.436 \\
AAO (mm) & $30.08 \pm 3.04$ & $30.00 \pm 3.05$ & 0.337 \\
PA (mm) & $19.77 \pm 3.27$ & $19.69 \pm 2.11$ & 0.943 \\
LV EDV (ml) & $96.08 \pm 25.36$ & $94.46 \pm 32.68$ & 0.807 \\
LV ESV (ml) & $32.92 \pm 16.62$ & $32.46 \pm 22.04$ & 0.943 \\
LV EF $(\%)$ & $65.85 \pm 10.89$ & $66.15 \pm 10.21$ & 0.929 \\
AV $(\mathrm{m} / \mathrm{s})$ & $1.35 \pm 0.20$ & $1.40 \pm 0.29$ & 0.495 \\
E peak $(\mathrm{m} / \mathrm{s})$ & $0.71 \pm 0.33$ & $0.69 \pm 0.21$ & 0.842 \\
e'(cm/s) & $6.38 \pm 2.93$ & $6.62 \pm 3.10$ & 0.785 \\
E/e' & $0.13 \pm 0.72$ & $0.11 \pm 0.03$ & 0.547 \\
TAPSE $(\mathrm{mm})$ & $20.08 \pm 3.69$ & $19.69 \pm 3.20$ & 0.706 \\
TDI-s $(\mathrm{cm} / \mathrm{s})$ & $9.77 \pm 4.57$ & $10.31 \pm 4.37$ & 0.470
\end{tabular}




\begin{tabular}{llll}
\hline & Baseline $(\mathrm{n}=13)$ & Follow-up $(\mathrm{n}=13)$ & $P$ value \\
\hline IVC $(\mathrm{mm})$ & $16.15 \pm 4.08$ & $17 \pm 2.83$ & 0.523 \\
Collapse rate $>50 \%(\mathrm{n} / \%)$ & $10(77 \%)$ & $11(85 \%)$ & 0.618 \\
LAEF $(\%)$ & $39.98 \pm 6.36$ & $44.67 \pm 5.01$ & 0.185 \\
\hline
\end{tabular}

LV indicates left ventricular; LA left atrium; RV right ventricular; RA right atrium; IVS interventricular septum; LVPW left ventricular posterior wall; AAO ascending aorta; PA pulmonary artery; EDV enddiastolic volume; ESV end-systolic volume; EF ejection fraction; AV aortic blood flow velocity; E peak mitral inflow early filling velocity; e', early diastolic myocardial velocity; TAPSE Tricuspid annulus systolic displacement; TDI-s early systolic myocardial velocity; IVC inferior vena cava; ${ }^{*}$ compared with control group , $P<0.05$; \# compared with mild patients, $P<0.05$.

Table 6 LAGLS, LV GCS and LVGLS in severe patients at baseline and follow-up

\begin{tabular}{llll}
\hline & Baseline $(\mathrm{n}=13)$ & Follow-up $(\mathrm{n}=13)$ & $P$ value \\
\hline LA GLS & $14.17 \pm 4.04$ & $18.36 \pm 4.89$ & 0.084 \\
LV GCS & $23.05 \pm 8.56$ & $30.40 \pm 7.51$ & 0.042 \\
LV GLS & $16.47 \pm 5.23$ & $19.78 \pm 8.11$ & 0.068 \\
\hline
\end{tabular}

LV indicted left ventricular; LA left atrium; EF ejection fraction; GLS global longitudinal strain; GCS global circumferential strain. ${ }^{*}$ compared with control group, $P<0.05$; \# compared with mild patients, $P<0.05$
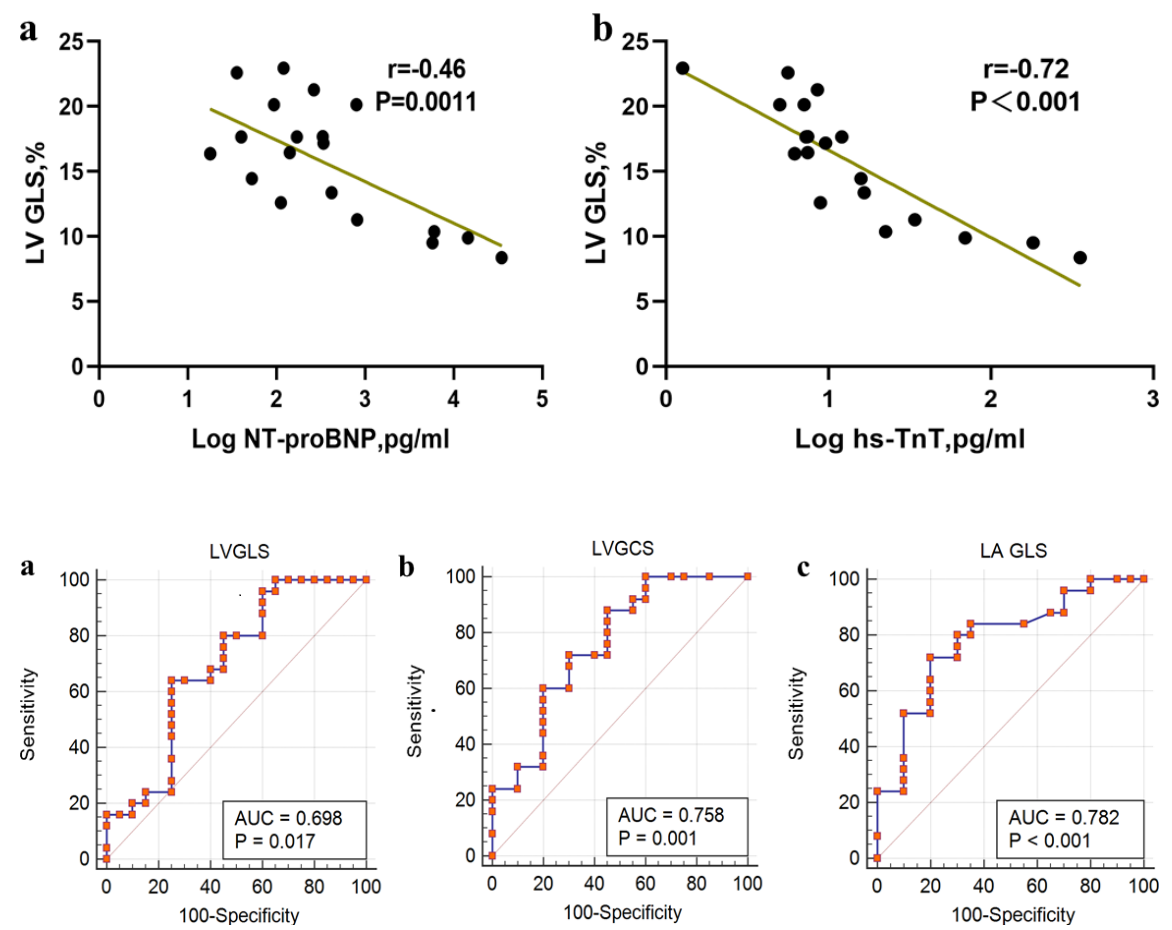

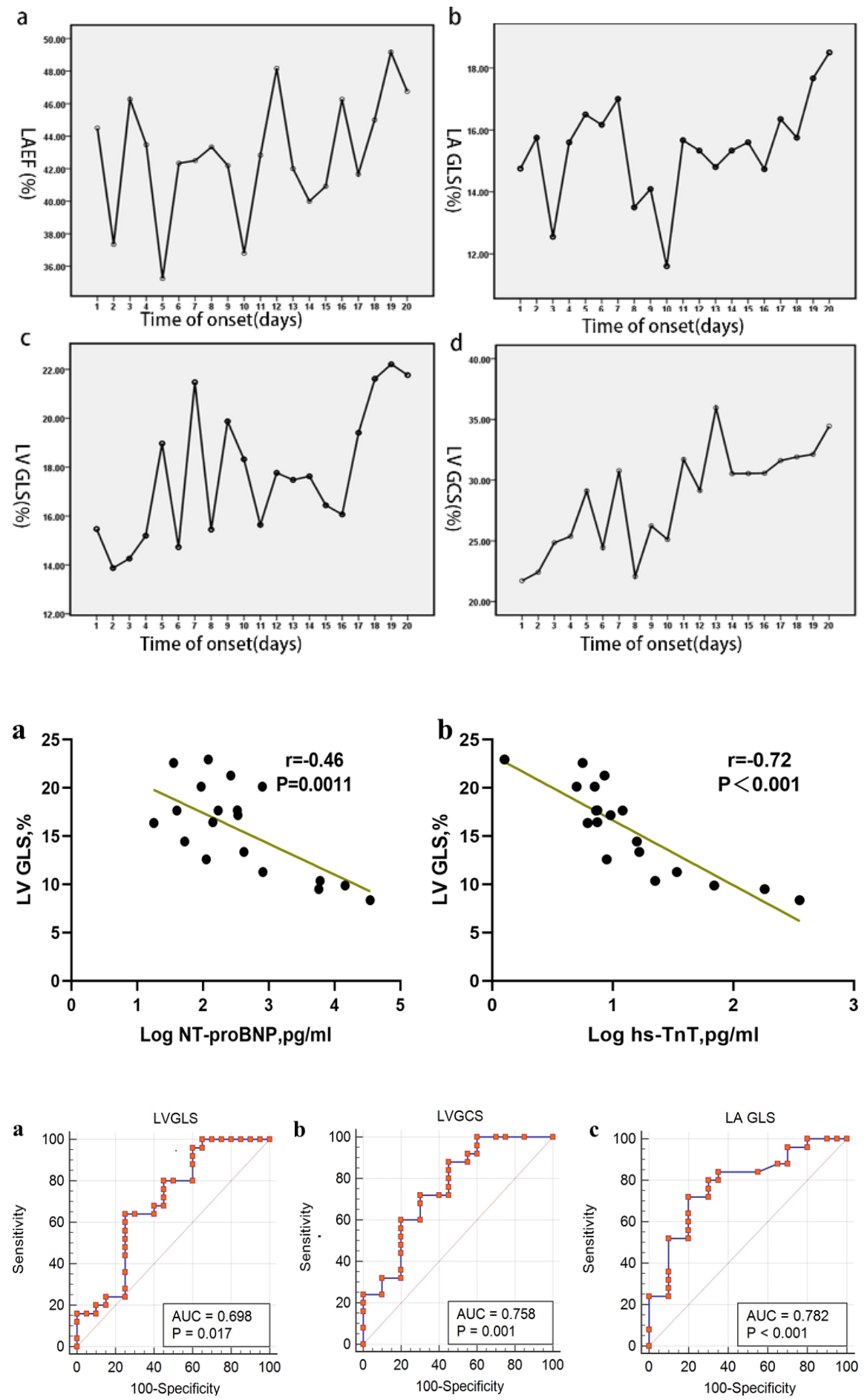

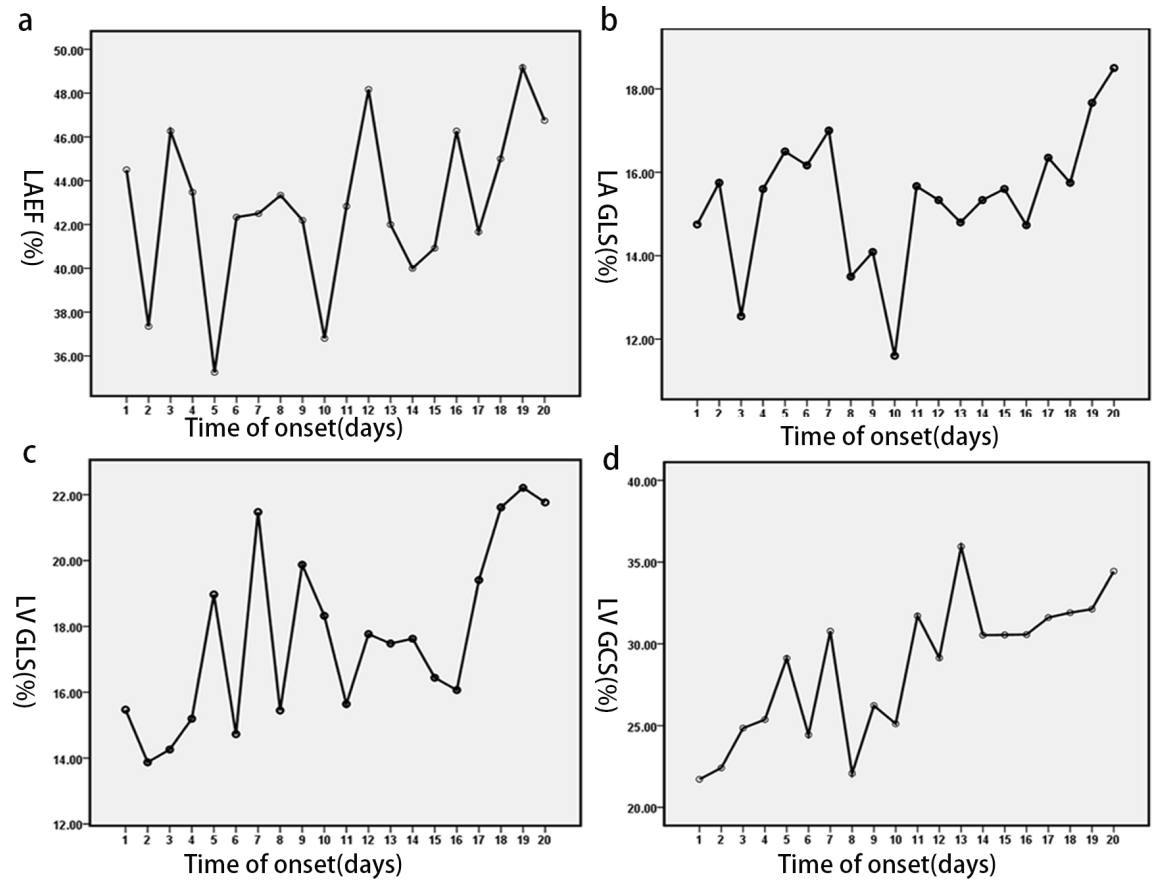